\title{
La moda en las sociedades avanzadas
}

\author{
Ana M artínez Barreiro \\ Universidad de la C oruña. La C oruña. Spain
}

\section{Resumen}

Este trabajo intenta desvelar el nuevo papel de la moda en las sociedades avanzadas, frente a la posición ideológica de la escuela francesa, integrada por Bourdieu y Baudrillard, aparecen otras posturas más centradas en la postmodernidad como las de Lipovetsky, M orace y $M$ affesoli que abordan el papel de la moda de forma diferente. Ya que el consumo jerárquico de la moda ha sido sustituido por el placer y la comodidad, pasando del «estatus símbolo» al «estilo símbolo» y al desencadenamiento del proceso tribal en el ámbito de las apariencias. El interés de este trabajo arranca, precisamente, de esta nueva perspectiva que intenta descifrar el nuevo papel de la moda en las sociedades postmodernas.

Palabras clave: consumo, moda, distinción, estética, postmodernidad, proceso tribal, individualismo, diferenciación..

\section{Abstract. Fashion in advanced soci eties}

This work attempts to reveal the new role of fashion in advanced societies, facing the ideological position of the French school, integrated for Bourdieu and Baudrillard, appear other postures more focused on post-modernity as the ones of Lipovetsky, M orace and $M$ affesoli; that broach the role of fashion a different way. Since the hierarchical consumption of fashion has been replaced for pleasure and comfort, going from the «status symbol» to the «style symbol» and also to the development of the tribal process in the field of the appearances. The interest of this work comes, precisely, from this new perspective that tries to decipher the new role of fashion in post-modern societies.

Key words: consumption, fashion, distinction, aesthetic, post-modernity, tribal-process, individualism.

\section{Sumario}

1. Introducción

2. La función social del objeto signo
3. La era del look

4. Conclusión 


\section{Introducción}

D esde que la sociedad de consumo de masas tuvo una presencia relevante en el campo de las ciencias sociales, han sido muchos los estudios realizados con la intención de desvelar el papel de la moda en las sociedades modernas. Vale como muestra la posición ideológica fundamental mente representada por la escuela francesa e integrada por Pierre Bourdieu y Jean Baudrillard, donde uno de los conceptos básicos que la caracterizan es el de la diferenciación.

Sin embargo, con el tiempo han surgido posturas cada vez más complejas y ambiguas producto sobre todo del «individualismo», de la «multidimensionalidad» y del «multiculturalismo» que caracterizan a la nueva sociedad de consumo de masas. $D$ e ahí que al gunos autores centrados en la postmodernidad, como Lipovetsky, M orace y M affesolí, aborden el papel de la moda de forma diferente, donde ya no solo tiene cabida el consumo jerárquico de la moda, sino que éste ha sido sustituido por el placer y la comodidad: pasando del estatus-símbolo al estilo-símbolo, como al desencadenamiento del proceso tribal en el ámbito de la apariencia. El interés de este trabajo arranca precisamente de esta nueva perspectiva que intenta descifrar el nuevo papel de la moda en las sociedades avanzadas.

\section{La función social del objeto signo}

Gran parte de las reflexiones sobre la moda hasta Thorstein Veblen aparecen incompletas y fragmentadas. Sólo a partir del año 1968 se manifiesta un interés más riguroso hacia los fenómenos colectivos como el de la moda. A esta atenta reflexión han contribuido los estudios sobre la sociedad de consumo de Jean Baudrillard y de Pierre Bourdieu. El mérito de Baudrillard consiste en haber colocado en un solo cuadro explicativo los fenómenos que normal mente han sido analizados singularmente. En efecto, sus obras principales: La sociedad de consumoㄹ , El sistema de los objetos ${ }^{2}$ y La economía política del signo ${ }^{3}$, siguen siendo el punto de partida obligado de toda reflexión respecto de la moda en la sociedad contemporánea.

En la base de sus análisis hay un esfuerzo por desmitificar la ideología del consumo como un comportamiento utilitarista de los sujetos condicionado por el goce y la satisfacción de sus deseos. Así, hoy en día la lógica social que condiciona el conocimiento operativo de los objetos de acuerdo con las diversas clases o categorías, tiene que ser al mismo tiempo un análisis crítico de la ideología del consumo. Este doble análisis, el de la función social distintiva de los objetos y el de la función política de la ideología que con ella se relaciona, se basa en una condición previa: la superación de la visión espontánea de los

1. BAU D RILLARD, J. (1974). La sociedad de consumo: susmitos, sus estructuras. Barcelona: Plaza $\&$ Janés.

2. BAUd RILLARD, J. (1969). El sistema de los objetos. M éxico: Siglo XXI.

3. BAUd RILLARD, J. (1974). La economía política del signo. M adrid: Siglo XXI. 
objetos en términos de necesidad o de su valor de uso. D e ahí, que la hipótesis consistente en asignar a los objetos un único estatus funcional sea falsa. Lo fundamental en las sociedades modernas es el valor de cambio signo. En efecto, «una verdadera teoría de los objetos y del consumo se fundará no sobre una teoría de las necesidades y de su satisfacción, sino sobre una teoría de la prestación social y de la significación»4.

Esta función primordial de los objetos se encuentra en Veblen bajo la noción de «derroche ostensible», cuya meta era significar el rango social. Y es siempre en aquello que tienen de fútil, de superfluo, de no funcional, esto es, en el juego de la moda, donde los objetos adquieren su significación de prestigio, designando al ser y la categoría social de su poseedor. Por ello, la teoría de Veblen ${ }^{5}$ se ha convertido en un marco de referencia y ha adquirido un valor de modelo interpretativo para entender el consumo como una estructura de segregación y de estratificación.

Para Baudrillard nunca se consume un objeto por sí mismo o por su valor de uso, sino en razón de su valor de cambio, es decir, en razón del prestigio, del estatus y del rango social que confiere. Pues, por encima de la satisfacción de las necesidades, hay que reconocer en el consumo de moda un instrumento de la jerarquía social, y en los objetos un ámbito de producción social de diferencias y val ores clasistas. La sociedad de consumo, con sus obsolescencias orquestadas, sus marcas más o menos cotizadas, no es más que un inmenso proceso de producción de valores signos cuya función es otorgar connotación a los rangos y reinscribir las diferencias sociales en una época igualitaria que destruye las jerarquías de nacimiento. Al efecto, la ideología hedonista que sustenta el consumo no es sino la coartada de una determinante más fundamental, la lógica de la superdiferenciación social. Pues la carrera del consumo y el afán de novedades no encuentran su fuente en la motivación del placer, sino que opera bajo el impulso de la competición de clases.

Ante semejante problemática, lo que motiva básicamente a los consumidores no es el valor de uso de las mercancías, sino que a lo que aspiran es al rango y a la diferencia social, pues los objetos no son más que exponentes de clase que funcionan como signos de movilidad y aspiración social. Precisamente, es la lógica del objeto signo la que impulsa a la renovación acelerada de los objetos y su reestructuración bajo el imperio de la moda, pues el fin de lo efímero y la innovación sistemática es reproducir la diferenciación social. Las audaces y aberrantes novedades de la moda tienen como función volver a crear distancias, excluir a la mayoría incapaz de asimilarlas de inmediato y distinguir, por el contrario, a las clases privilegiadas que sepan apropiárselas. En este sentido, «la función social de la innovación formal en materia estética es una función de discriminación cultural. Ya que la innovación formal en materia de objetos no tiene como fin un mundo de objetos ideal, sino un ideal social,

4. BAUDRILLARD, J. Ibídem, p. 2.

5. Veblen, T. (1974). Teoría de la clase ociosa. M éxico: Fondo de Cultura Económica. 
el de las clases privilegiadas, que es el de reactualizar perpetuamente su privilegio cultural»6.

Lo nuevo en moda es ante todo un signo distintivo, un «ujo de herederos» que, lejos de acabar con las disparidades sociales frente a los objetos, «la moda, como la cultura de masas, se dirige a todos para volvernos a poner a cada uno en su lugar. Es una de las instituciones que mejor restituye y cimienta, so pretexto de abolir, la desigualdad cultural y la discriminación social»7. Aún más, contribuye a la inercia social por cuanto la renovación de los objetos permite compensar una ausencia de movilidad social real. En este sentido, la moda como instrumento de distinción de clases, reproduce la segregación social y cultural, y participa de las mitologías modernas que enmascaran una igualdad inexistente.

O tro punto obligado de reflexión teórica en las sociedades contemporáneas es la teoría de la distinción8, donde se ejemplifica el capital cultural como generador de estilos de vida diferenciados. En este sentido, Pierre Bourdieu evoca que cada uno de los universos de preferencias que existen, al funcionar como un sistema de variaciones diferenciales, permiten expresar las diferencias sociales de forma tan completa como los sistemas más refinados. «Y es, porque la relación de distinción se encuentra inscrita en él y se vuelve a activar, en cada acto de consumo, mediante los instrumentos de apropiación económicos y culturales que se exigen »9. D e ahí que la posesión de ciertos bienes culturales atestigüen no sólo la riqueza de su propietario, sino también su buen gusto, como una garantía de legitimidad. Pues, de la misma forma que los bienes culturales están sutilmente jerarquizados, para marcar los grados de progreso iniciático, los beneficios de distinción están destinados a deteriorarse si el campo de producción, regido por la dialéctica de la pretensión, no ofreciera continuamente nuevos bienes o nuevas maneras de apropiársel os ${ }^{10}$.

Sin embargo, en materia vestimentaria el ajuste entre la oferta y la demanda no está en los condicionamientos de la producción, ni en el sometimiento de ésta a los gustos de los consumidores, sino, como dice Bourdieu, en «la correspondencia de dos lógicas relativamente independientes, la lógica de los campos de producción y la lógica del campo del consumo»11. Este principio de la homología funcional y estructural hace que la lógica del campo de producción y la lógica del campo de consumo sean concertadas de manera objetiva, por el hecho de que todos los campos especializados como el del vestido tienden a organizarse según el mismo criterio12. D e ahí que el acuerdo que se establece

6. Ibídem, p. 28.

7. Ibídem, p. 34.

8. BouRdieu, P. (1991). La distinción, criterio y bases sociales del gusto. M adrid: Taurus.

9. Ibídem, p. 223.

10. El ensayista inglés $\mathrm{H}$ irsch trasladó este tipo de análisis a los bienes económicos para explicar la ferocidad de la competencia en el capitalismo a raíz de la lucha desenfrenada por los bienes posicionales.

11. BouRdieu, P. Ibídem, p. 228.

12. Según el volumen del capital específico poseído y según la antigüedad de la posesión. Ibídem, p. 230. 
entre unas clases de productos y unas clases de consumidores no se realiza más que por mediación de esa especie de la homología entre unos bienes y unos grupos que definen el gusto: elegir según los gustos propios es delimitar unos bie nes concebidos según su posición, armonizados entre sí y ayudados a su vez por unas instituciones que se rigen por el mismo principio. En resumen, las incesantes transformaciones de la moda son producto del concierto objetivo, por una parte, de la lógica de las luchas internas en el campo de producción que se organizan según la oposición entre lo antiguo y lo nuevo, lo caro y lo barato... , y por otra, lo lógica de las luchas internas en el campo de la clase dominante que enfrentan a los poseedores y a los pretendientes pretenciosos.

En lugar de un espacio social subjetivista, Bourdieu llega a construir el espacio social en un sentido objetivo13, es decir, con una estructura de relaciones objetivas que determina la forma que pueden tomar las interacciones y la representación que de ellas pueden tener aquéllos que se encuentran en dicho espacio. D e ahí que las posiciones sociales se presentan como unas plazas que hay que defender y conquistar, empleando estrategias para ello14. Ahora bien, no es casual que la búsqueda de la distinción no necesite presentarse y afirmarse como tal, pues una educación burguesa basta para determinar los cambios más especiales en un momento dado del tiempo. Por el contrario, la pequeña burguesía de formación reciente hace demasiado, traicionando así su inseguridad, y se desvaloriza por la propia intención de distinción15. Lo que significa que las estrategias de pretensión estén perdidas de antemano16. Lo que Bourdieu quiere decir es que la ilusión sociológicamente fundada de la distinción natural reposa en el poder que tienen los dominantes de imponer, con su existencia misma, una definición de la excelencia que, al no ser otra cosa que su propia manera de existir, está destinada a presentarse, a la vez, como distintiva y natural.

\section{La era del look}

Los elementos conceptual es propuestos están muy lejos de agotar las posibilidades de análisis del fenómeno de la moda. D e hecho, como consecuencia

13. Bourdieu elabora un espacio social estructurado en base a los tres componentes de la clase social: «capital económico», «capital cultural» y «capital social». Los agentes tienen sobre este espacio social unos puntos de vista que depende de la posición que en el mismo ocupan, y en los que a menudo se expresa su voluntad de transformarlo o conservarlo. Ibídem, p. 169.

14. Ibídem, p. 241.

15. Ibídem, p. 246.

16. Elster, J. (1988). U vas amargas. Barcelona: Península, p. 99-106. Este autor hace una crítica a Bourdieu, por el carácter contradictorio de las estrategias de distinción (conductas premeditadas para tratar de impresionar), y hace notar que hay consecuencias futuras 0 imprevistas que no pueden obtenerse de una estrategia premeditada, sino como una consecuencia no querida o imprevista, esto es, como un subproducto de otras conductas ajenas llevadas a cabo para otros propósitos independientes. 
del apogeo del individualismo contemporáneo ha emergido una nueva línea de investigación que podría ser definida como la perspectiva ecológica de la moda y que está fundamental mente representada por Li povetsky, M orace y $M$ affesolí. En efecto, tras la crisis de las utopías revolucionarias y la caída de los colectivismos asistimos hoy a una revitalización del individualismo que conlleva, entre otros efectos importantes, un desplazamiento del interés de lo público a lo privado y un ensalzamiento de los valores asociados a éste último.

Esta mudanza, que es definida por Lipovetsky como la «segunda revolución individualista» 17 , se traduce en el declive de la moral centrada en el deber y su transformación privada del individuo18. En este sentido, los valores permisivos, hedonistas y psicologistas relevan a los valores disciplinarios y rigoristas, que eran los dominantes en la cultura del industrialismo burgués hasta el desarrollo del consumo y la comunicación masiva. En efecto, el individualismo narcisista se convierte en el nuevo trasfondo moral de las sociedades contemporáneas.

En el ámbito de la moda, uno de los cambios que testimonian la emergencia de personalidades narcisistas, propias del individualismo contemporáneo, es que, paral elamente a la apariencia legítima, aparecen nuevos comportamientos individuales y colectivos en ruptura con el momento anterior. Si durante mucho tiempo la lógica social de la moda fue la de distinguirse socialmente mediante la manera de vestir, con el surgimiento del prêt-à-porter y el fin de las tendencias de temporada marcadamente unificadas se vaticina el final del dirigismo disciplinario de la apariencia y la aparición de la multiplicidad estética. Ya no hay una «sola moda», sino «una multiplicidad de modas» igualmente legítimas ${ }^{19}$. Paralelamente, frente a esa fragmentación estilística, se producen otros cambios muy significativos en los comportamientos y en las motivaciones del individuo frente a la moda.

En primer lugar, se constata una mayor autonomía de los consumidores en relación con las novedades. M ientras en la fase anterior había que adoptar los últimos modelos lo más rápidamente posible, en la actualidad, por el contrario, la tendencia es meramente indicativa. Ya no hay una correspondencia entre la innovación y la difusión, entre la vanguardia creativa y el público consumidor, pues «la calle» se ha emancipado de la fascinación de los líderes de la moda y asimila las novedades a su ritmo y a su antojo. Así, es muy difícil estar absolutamente pasado de moda. En segundo lugar, cuando ya no hay

17. LipovetSky, G. (1986). La era del vacío. Barcelona: Anagrama, p. 9.

18. Ésta es la tesis que Lipovetsky ilustra en su libro El crepúsculo del deber. Barcelona: Anagrama, 1994.

19. Sobre estos temas, véase, LIPOVETSKY, G. (1980). «La M oda abierta». En El imperio de lo efímero. Barcelona: Anagrama, p. 119-171. Y «La era del look. La bal canización de la moda: libertad y ansiedad de las apariencias». M adrid: El País, jueves 18 de noviembre de 1993. También, M ORACE, F. «Tendencias del comportamiento de la moda en I talia». Sevilla: Universidad Internacional M enéndez Pelayo, septiembre de 1988, p. 127-138. Contratendencias. M adrid: Ed. C eleste, 1993. 
moda unitaria «el look funciona a la carta»20. Las mujeres continúan siguiendo la moda, pero de manera más libre. Llevan lo que les gusta, lo que les va, no la moda por la moda. El mimetismo directivo característico de la moda clásica ha dado paso a un mimetismo de tipo optativo. Se imita lo que se quiere, cuando se quiere y como se quiere. En tercer lugar, este individualismo narcisista conduce a la relajación de la preocupación por la moda. La moda entra en la era desapasionada del consumo, en la era de la curiosidad relajada y diversificada. La lógica cool ha invadido el espacio de la moda y no sólo el de la escena política. En cuarto lugar, otro de los efectos más importantes del individualismo contemporáneo respecto a la moda es que ha reducido la dimensión del símbolo jerárquico en favor del placer, la comodidad y la libertad. Pues a través del vestido ya no se busca prioritariamente hacer alarde de pertenencia a una clase social, sino de un gusto, de un estilo de vida. En este punto, Ios análisis de la sociología moderna, representada hoy por Bourdieu y Baudrillard, parecen haber olvidado una parte esencial de la explicación de la moda contemporánea al haber permanecido ciegos a un nuevo tipo de regulación social cuya base es la seducción y la hiperelección.

Ahora bien, tanto Lipovetsky como M orace no niegan que los objetos puedan ser significantes sociales y signos de aspiración social, «lo que cuestionan es la idea de que el consumo de masas se rija exclusivamente por el proceso de distinción y diferenciación clásica» 21 , pues en las sociedades modernas se ha desencadenado un proceso de desocialización del consumo y de regresión de la primacía inmemorial de los valores clasistas de los objetos en provecho del valor dominante del placer individual y del objeto uso. Así, el consumo de moda ha dejado de ser una actividad regulada por la búsqueda de reconocimiento social para desplegarse hacia el bienestar, la funcionalidad y el placer en sí mismo. D e este modo avanza el individualismo narcisista, al cual corresponde no sólo el desarrollo del furor psíquico y corporal, sino una nueva relación con los demás y con las cosas. La era de las motivaciones íntimas y existenciales, de la gratificación psicológica, del placer por sí mismo, de la calidad y de la utilidad de las cosas, ha tomado el relevo. Todo esto contribuye a adoptar una perspectiva muy distinta acerca del papel de la moda. Lejos de aparecer como un vector de reproducción de las diferencias sociales, el sistema de la moda en expansión ha permitido, más que cualquier otro fenómeno, la continuidad de la trayectoria secular hacia la conquista de la autonomía individual y la reducción de las distancias sociales.

Frente al individualismo que rechaza cualquier consideración de orden social al devenir de la moda, ha surgido otra corriente del pensamiento que, sin reproducir los patrones clásicos de la distinción de la moda, cuestiona el papel exclusivo del yo y realza la importancia de la extensibilidad del yo en tribus a las que se pertenece simultáneamente o sucesivamente. Ésta es la hipó- 
tesis que M affesolí extiende en su libro L os tiempos de las tribus22, al asegurar que nuestro fin de siglo exhibe una sociabilidad caracterizada por una dialéctica constante entre la masificación creciente y el desarrollo de unos microgrupos llamados tribus.

En este sentido, para poder reflexionar sobre el devenir de la moda hay que aclarar la idea de por qué hoy asistimos al reemplazamiento de una identidad estable por un portador de máscaras sucesivas en esferas sociales cambiantes. En relación con la persona, hay que llamar la atención sobre la existencia de que tenemos una multiplicidad de yoes, en cuanto que en cada momento, «soy otro». Por consiguiente, lo que caracteriza y lo que marca nuestro tiempo es la estética como expresión efectiva del ambiente tribal23. Esta idea de tribu, hace que estemos entrando en un tipo de sociedad en el que va a dominar la idea de extensibilidad del yo, un ego que se pierde en la tribu24. Cuando uno se viste de la misma manera que se viste su tribu, ya no tiene, propiamente hablando, un sólo ego: hay aquí un ego extensible. Cuando uno lleva el uniforme punk, cuando uno lleva el traje burgués de tres piezas, se pierde en su tribu; y si en otro momento del día lleva otro uniforme (cambia, por ejemplo, su traje clásico por el pantalón tejano, o por cualquier otro vestido de sport) entra en otra tribu. D e ahí, que hay algo de extensible en mi identidad, pues no soy únicamente estable, me pierdo mediante la idea de extensibilidad del yo.

Si durante mucho tiempo uno de los grandes conceptos básicos que había caracterizado a la sociedad moderna era el concepto de separación, tal como ha sido formulado por la tradición sociológica francesa, M affesolí piensa que este concepto de separación y de distinción está superado y lo que se aplicaba a la modernidad es la integración emocional, a través del paradigma estético. En este paradigma estético, la mezcolanza vestimentaria (los cabellos multicolores, la manera de reutilizar los vestidos retro) tiene, en cierto sentido, la función de cemento, de unirme a los otros.

En este sentido, la apariencia, el retorno de la imagen a un primer plano, va a tener una función de tipo comunitario. Pero se trata de un sentimiento y de una experiencia compartida que hacen que sea siempre en el interior de un grupo donde yo experimente algo y donde tenga que expresarme. Esto le lleva a decir a M affesolí que no hay narcisismo en las sociedades postmodernas, sino más bien una especie de sujeto colectivo, una extensibilidad del yo, que aparece particularmente representada en especies de reagrupamientos de tipo puntual. Las grandes ciudades modernas nos ofrecen buenos ejemplos de estas agrupaciones cuando uno observa en la vida cotidiana la representación en público de la sucesión de clichés (del jogging, del punk, del look retro o la moda grunge, etc.). Todo esto da buena cuenta de este proceso tribal, que es el proceso

22. M AfFeso lí, M . (1990). Los tiempos delas tribus. El dedive del individualismo dela sociedad de masas. Barcelona: I caria.

23. M AfFeso Li, M. «Los juegos y las máscaras: modas y tribus en la postmodernidad». Santander: Universidad M enéndez Pelayo, agosto de 1987, p. 92.

24. Ibídem, p. 94. 
de la modernidad. D e ahí la importancia de la apariencia que de modo internacional sellama look.

\section{Conclusión}

A modo de conclusión, podemos decir que diversas valoraciones sobre la moda han visto la fragmentación de clases, la consolidación de la importancia de la imagen y el triunfo del deseo como móvil del comportamiento individual, más aun en este final de siglo la moda se está orientando hacia formas más maduras y sutiles creándose un mecanismo de autorregulación mediante el cual los consumidores eligen los productos en términos de ecología, rechazando con decisión la lógica de la diferenciación y buscando en su vestimenta la lógica de la identidad profunda. 